\title{
Computational Analysis of Mixing Characteristics of Jets from Rectangular Nozzle with Internal Grooves
}

\author{
M. Sundararaj ${ }^{* *}$ and S. Elangovan ${ }^{2}$ \\ 'Associate Professor, Department of Aeronautical Engineering, Bharath University, Chennai-600 073, India; \\ sundarsonic@yahoo.com \\ 2Professor, Department of Aeronautical Engineering, Bharath University, Chennai-600 073, India; \\ subelango@yahoo.co.in
}

\begin{abstract}
A Computational study has been carried out to understand jet flow development from plain and grooved rectangular nozzles of aspect ratio 2:1. Grooves of square cross section of side $4 \mathrm{~mm}$ and axial length $5 \mathrm{~mm}$ were introduced at the exit of the nozzle in three different orientations as (i) minor-axis, (ii) major-axis and, (iii) in both minor and major axes. The computational studies were carried out using computational software ANSYS CFX for a nominal jet exit velocity of $20 \mathrm{~m} / \mathrm{s}$. Velocity distribution along the axis of the jet is observed from computational results and compared with the available experimental results. Grooves seem to have very negligible effect on the near field region but significantly influence the jet decay in the far field.
\end{abstract}

Keywords: Passive Control, Jet Mixing Enhancement, Rectangular Jet and Center Line Velocity Decay.

\section{Introduction}

There are many engineering devices in which jet producing nozzles form an important component. Circular nozzles are most commonly used but non-circular nozzles are also used primarily due to their desirable mixing characteristics. They are found to exhibit enhanced entrainment of ambient fluid. In view of their favorable qualities many researchers have carried out extensive study of rectangular jets, both experimentally and computationally.

\section{Literature Survey}

Non circular jets have been found to spread and mix faster than circular jets. Ho and Gutmark [1] investigated flow characteristics of an elliptic jet with an aspect ratio of 2:1. The mass entrainment before the end of the potential core was found to be three to eight times than that in an axisymmetric or a two dimensional jet. Investigations have independently been carried out for different aspect-ratio elliptic jets. Hussain and Husain [2] carried out a comparative study of aspect ratio in the range of $2: 1-8: 1$. It was observed that for a given equivalent diameter, the aspect-ratio is an important parameter controlling the deformation and typological changes, that is, bifurcation of large-scale vortical structures in elliptic jets, and that the dynamics of low aspect ratio elliptic jet are basically different from that of jets of moderate aspect ratios.

Substantial efforts have been devoted to investigating the properties of jets emerging from non-circular nozzles. Verma and Rathakrishnan [3] studied elliptic nozzle with corners, e.g. rectangular, triangular, have observed that as the jet spreads, its cross-section can regularly evolve through shapes similar to those of the jet nozzle, but with axes successfully rotated at angles characteristic of the jet geometry, referred to as the axis-switching phenomenon. Srinivasan and Rathakrishnan [4] had done experiments

* Corresponding author:

M. Sundararaj (sundarsonic@yahoo.com) 
in polygonal slot jets and observed that mixing processes continues to be active in far field. This indicates that polygonal jets, particularly those of low orders, may be favorably considered for use in mixing and propulsive systems.

Elangovan and Rathakrishnan [5] studied the effect of internal grooves on nozzle on high speed jets. Experiments were carried out on jets issuing from circular nozzles with grooved exits and results were compared with that of plain nozzle. They found that the shock cell structure of under expanded jets from grooved nozzles appeared to be weaker than that of plain nozzle. The iso-Mach contours indicate that the jet spread along the groove plane is significantly higher than the plain nozzle; this was due to the stream wise vortices shed from the grooves. Arun Kumar et al. [6] carried out an experimental study of jets issuing from grooved rectangular nozzles of aspect ratio 2:1 using two component hot wire anemometer. They observed that grooves significantly influenced the jet development beyond the potential core region. S. Paul Pao [7] presents a unified method for subsonic and supersonic jet analysis using the three- dimensional Navier-Strokes code PAB3D. The Navier-Strokes code was used to obtain solutions for axisymmetric jets with on-design operating conditions at Mach numbers ranging from 0.6 to 3.0, supersonic jets with non-axisymmetric nozzle exit geometries.

The objective of the present study is to analyze the jet from grooved rectangular nozzles by numerical simulation and validate the results with the experimental results obtained by Arun Kumar et al [6].

\section{Computation}

\subsection{Geometry}

The Geometry required for the computations are developed using CATIA. A typical nozzle geometry developed by using CATIA along with the exit size of the nozzle is presented in Figure 1. The figure shows that the exit dimension of the Nozzles is $23.5 \mathrm{~mm}$ breadth (minor axis) and $47 \mathrm{~mm}$ height (major axis). The aspect ratio of the nozzle exit was maintained as 2 (length of major axis / length of minor axis). The length of the nozzle from inlet to exit is $300 \mathrm{~mm}$. Figure 2 shows the dimensions of computational domain along with the nozzle used for the present study. A square cross section of side $300 \mathrm{~mm}(8 \mathrm{~d})$ and $600 \mathrm{~mm}(16 \mathrm{~d})$ length was used as the computational domain in the study.

Four different orientation of nozzle exits were considered for the computation as described below. Figure 3 shows

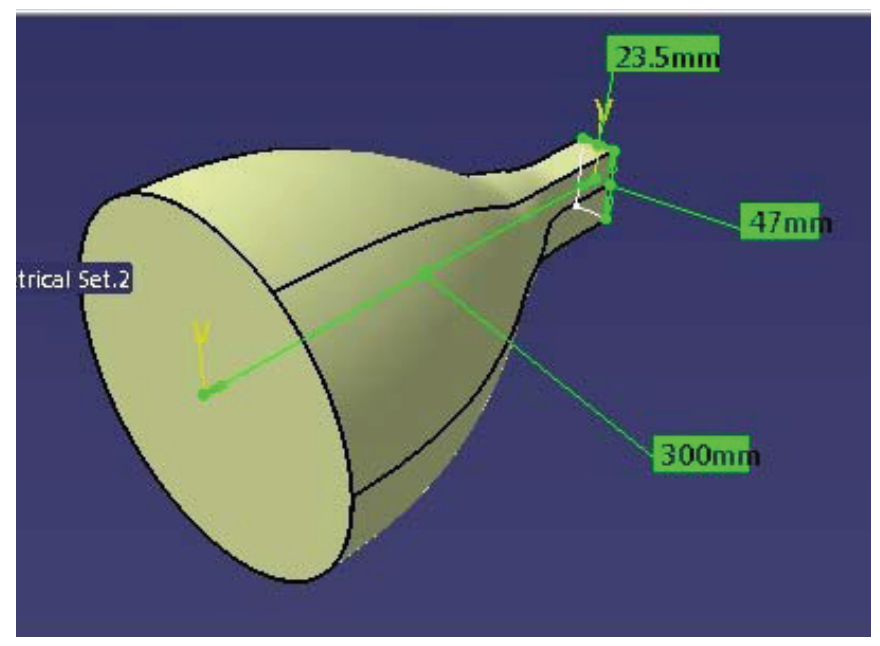

Figure 1. Dimensions of the nozzle.

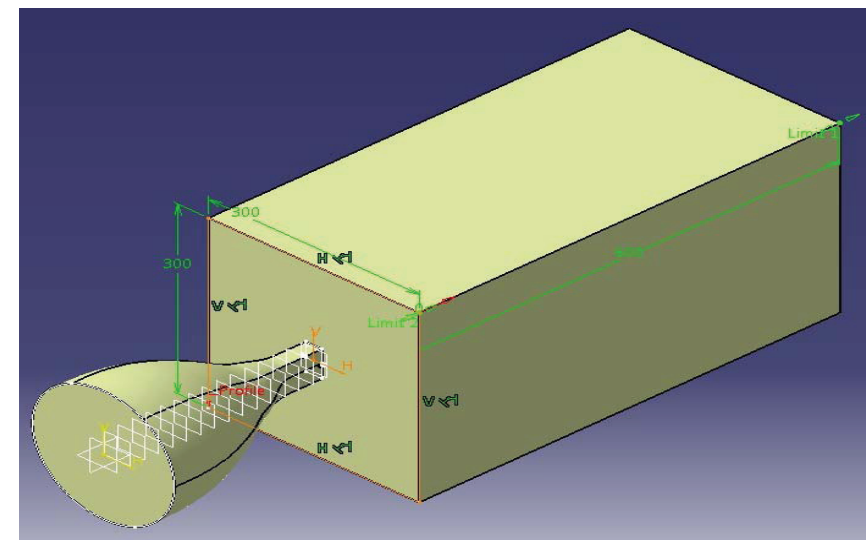

Figure 2. Dimensions of the computational domain with nozzle section.

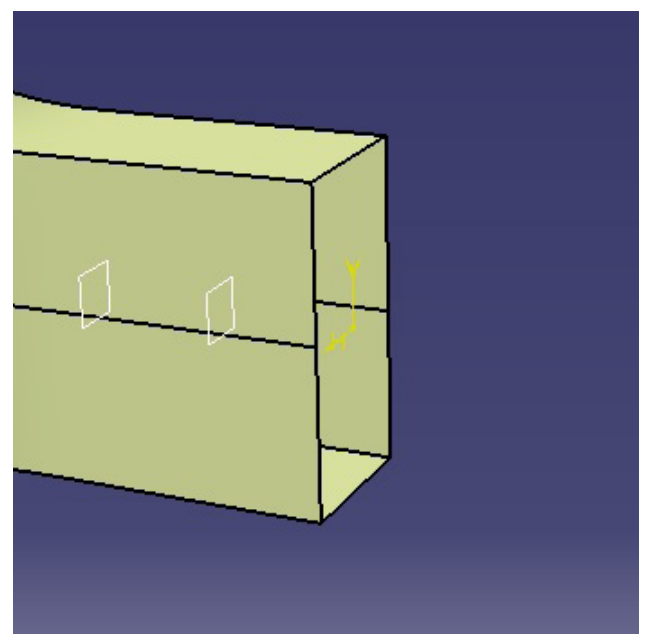

Figure 3. Exit shape of the plain nozzle.

the exit of the nozzle without any groves, where as the Figure 4 shows the presence of square groove of side $4 \mathrm{~mm}(0.107 \mathrm{~d})$ 


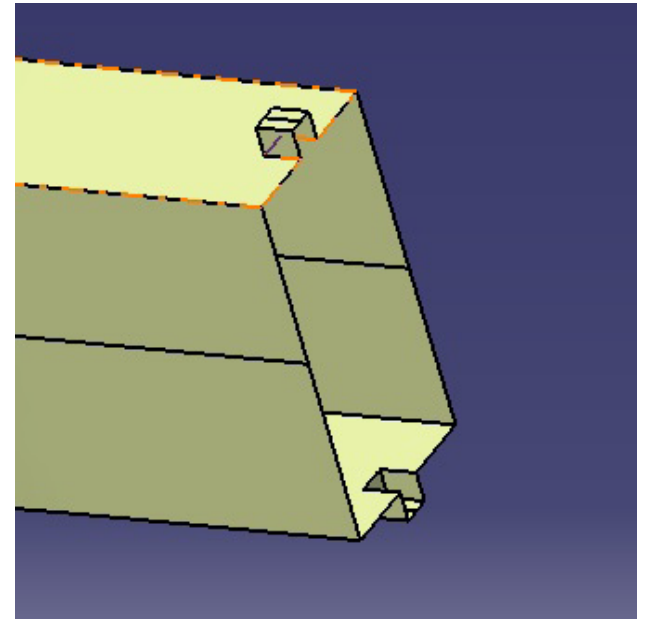

Figure 4. Nozzle exit with grooves in major axis.

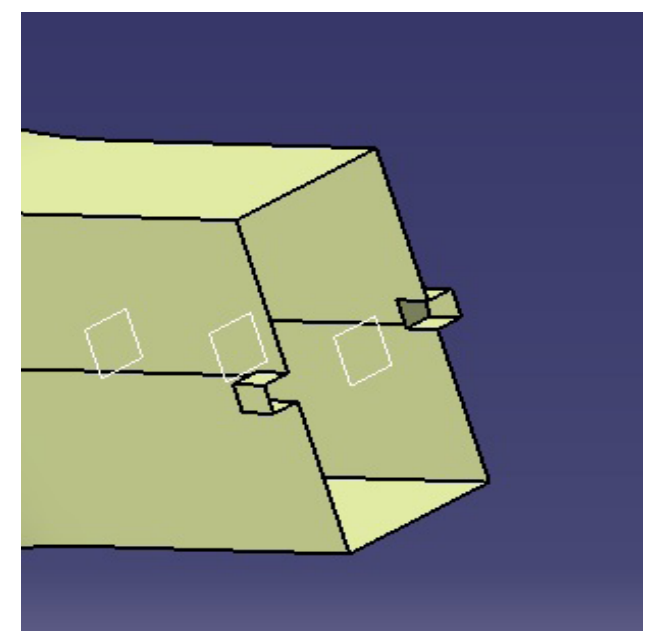

Figure 5. Nozzle with grooves in minor axis.

and length $5 \mathrm{~mm}$ (1.333d) at the major axis of the exit of the nozzle. Figure 5 and 6 shows the nozzle exit orientation with square grove at minor axis and at both the axis respectively.

\subsection{Mesh Generation}

Geometry of the different configuration nozzles along with computational domain developed using CATIA was used for the mesh generation in ANSYS ICEM CFD. For the mesh generation of all the geometry Global element seed size and scale factor are selected as 8 units and 1 respectively. Unstructured tetrahedral volumetric mesh was generated using the software. Since the velocity decay along the jet axis is primary interest, a dense mesh of maximum element seed size 2 units (mesh concentration) was generated near the potential core region. A typical cut section view of generated mesh along the vertical plane is shown in the Figure 7. Total number of

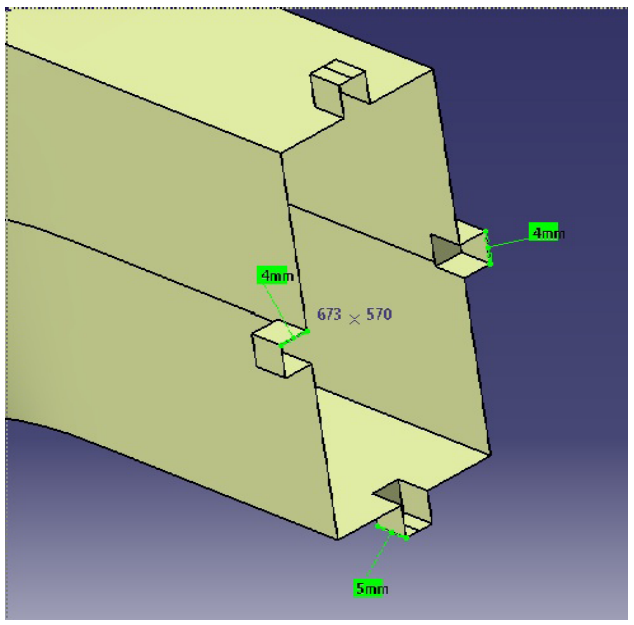

Figure 6. Nozzle with grooves in both major and minor axis.

elements and nodes for different nozzles was maintained approximately $24,00,000$ and 4,00,000 respectively.

\subsection{Boundary Conditions}

The mesh file generated using ICEM CFD was imported into ANSYS CFX where all the boundary conditions required for the computation are defined. Two importent boundary conditions (inlet of the nozzle and outlet of the domain) were defined based on the experimental data availabe in Arun kumar et. al. Reference pressure of all the computation was taken as the normal atmospheric pressure (101325 Pa). At the inlet of nozzle, velocity and temperature was defined as $0.683 \mathrm{~m} / \mathrm{s}$ and $302 \mathrm{k}$ respectively. Minimum turbulence level of $1 \%$ was set at the inlet boundary. Outlet of the domain defined as the opening with relative pressure of $0 \mathrm{~Pa}$ (since the domain exit is far away from the jet exit, that is $16 \mathrm{~d}$ ). Walls of the nozzle and doamin were defined as no slip condition with adiabatic condition. Some of the importent solver parameters used during the computations are give below.

- Turbulence model : k- $\varepsilon$ model

- Heat transfer model : Isothermal model

- Advection scheme : High resolution scheme

- Residual target : 0.0001 .

\section{Results and Discussion}

Effect of introduction of grooves at the exit of the nozzle on the flow field parameters were studied from computational results. Velocity decay along the axis of the jet for 


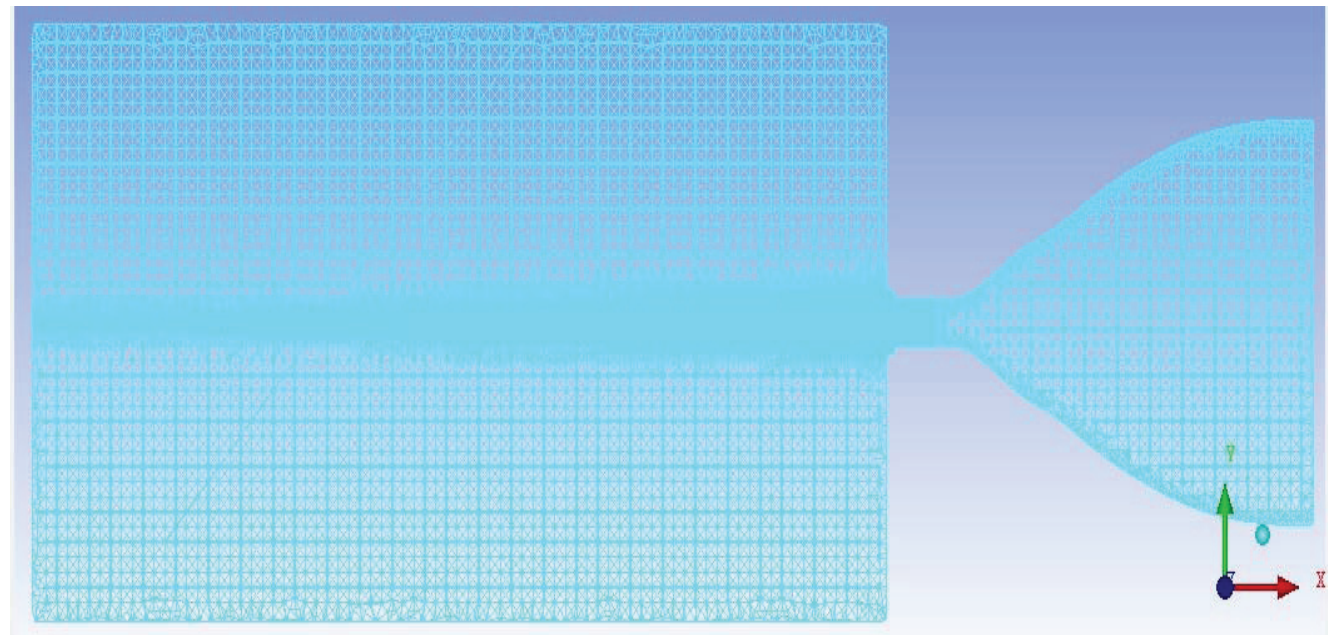

Figure 7. View of mesh cut plane along the vertical plane.

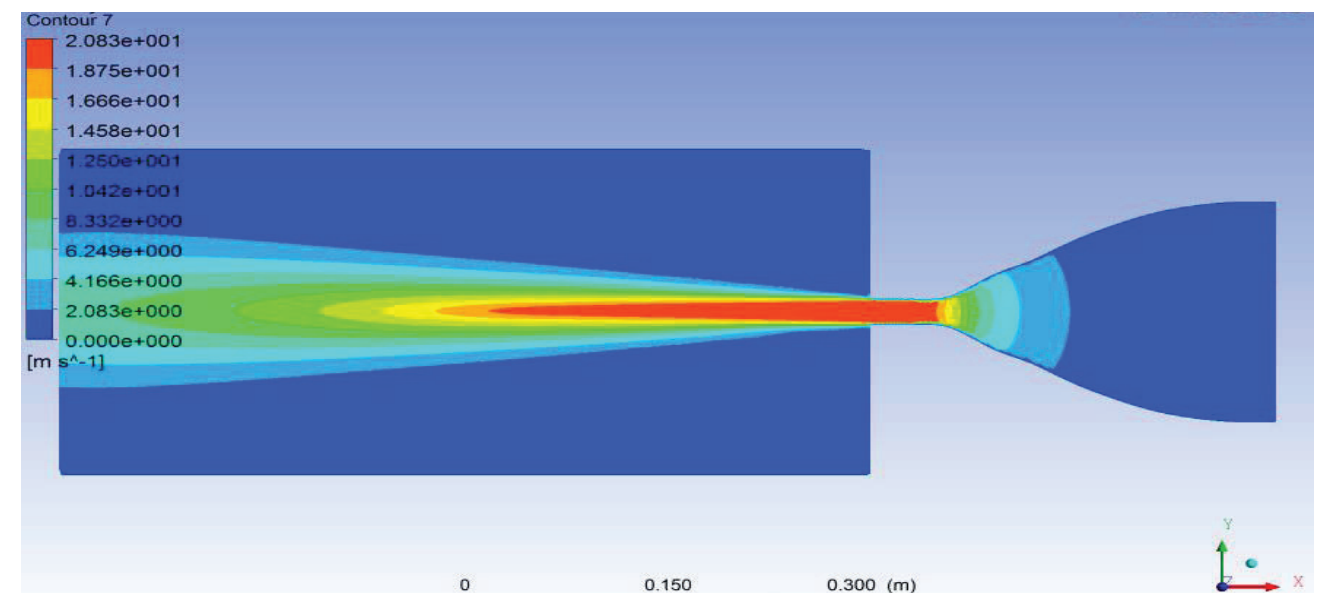

Figure 8. Contour plot of velocity distribution of Jets from Plain Jet (at $\mathrm{z}=0$ ).

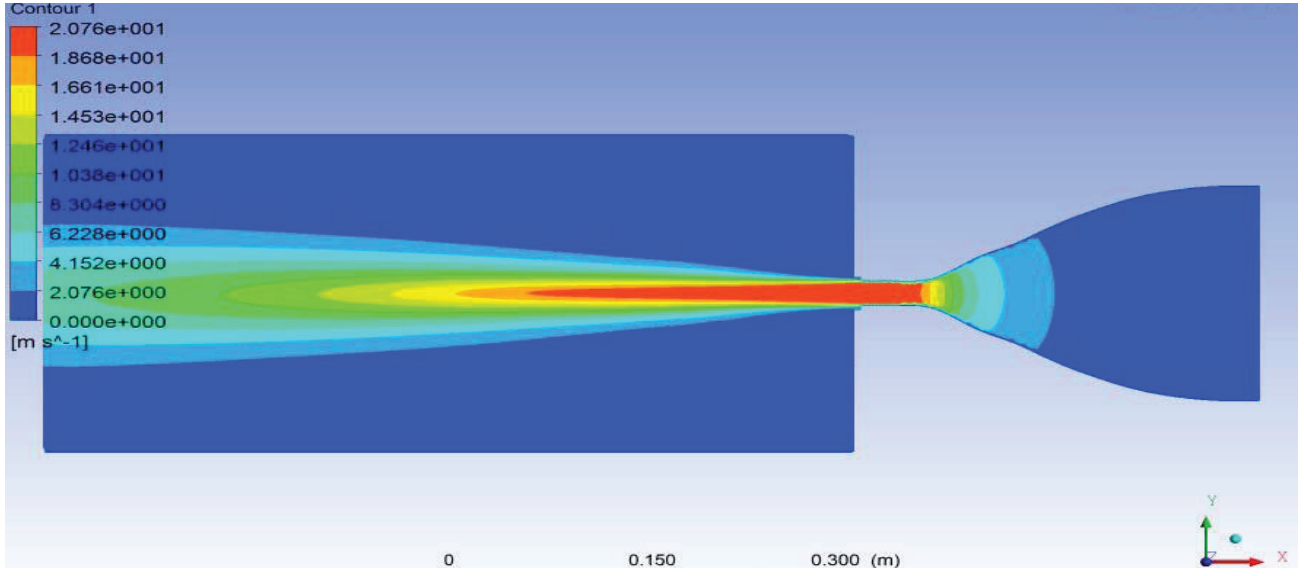

Figure 9. Contour plot of velocity distribution of Jets with grooves on both the axis $($ at $\mathrm{z}=0)$.

different configuration of groove arrangement obtained from computational results was compared with the available experimental results.
Figure 8 and Figure 9 shows the contour plot of velocity distribution of rectangular without and with grooves at the exit respectively. Figure clearly shows that the velocity 
at the exit of the nozzle is $20 \mathrm{~m} / \mathrm{s}$ (Red color), length of potential core of the jet and velocity distribution of the jet expansion. From the contour plot there is no significant difference in observation of the flow parameters of with and without groove case.

Mean velocity decay along the axis of rectangular jet with different jet exit configuration were measured at 20 different locations by Arun et.al ${ }^{6}$. The local centerline velocity $(\mathrm{U})$ is normalized by the exit velocity $\left(\mathrm{U}_{\mathrm{e}}\right)$ and is plotted against the normalized axial distance by the nozzle equivalent diameter $\left(\mathrm{D}_{\mathrm{e}}\right)$. Figures 10 to 13 , comparison of experimental and computational velocity decay of the different jet exit conditions were presented. For all the exit configurations there is no significant difference in velocity distribution of computational and experimental results between jet exit plane to potential core length $\left(0 \mathrm{D}_{\mathrm{e}}\right.$ to $\left.3.9 \mathrm{D}_{\mathrm{e}}\right)$. Downstream of potential

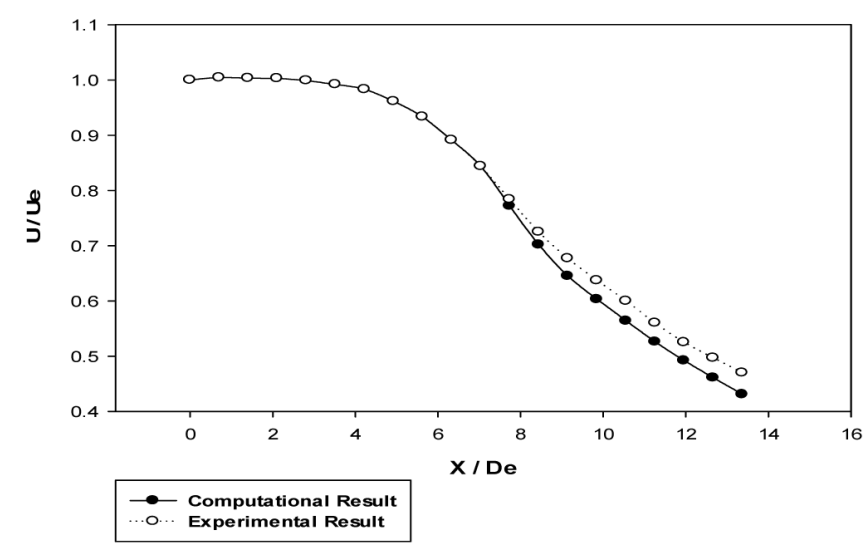

Figure 10. Centerline velocity decay of the jet without groove.

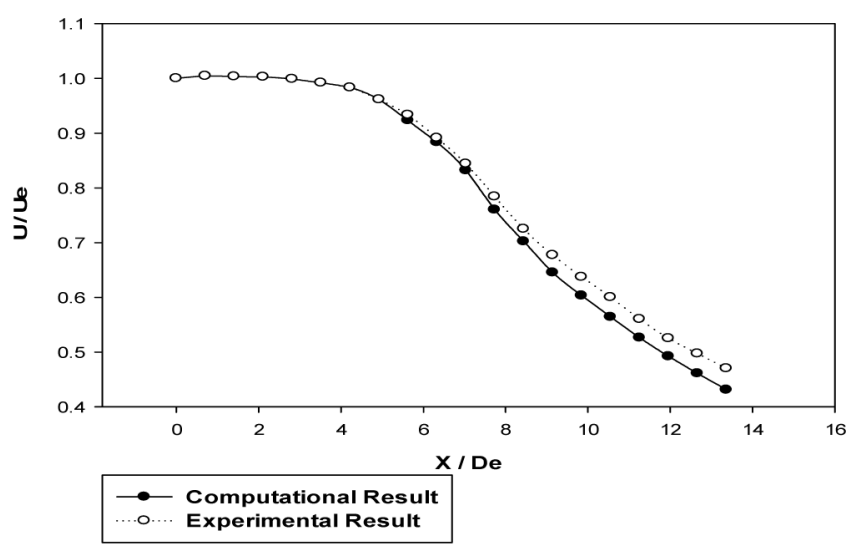

Figure 11. Centerline velocity decay of the jet with groove in Major Axis.

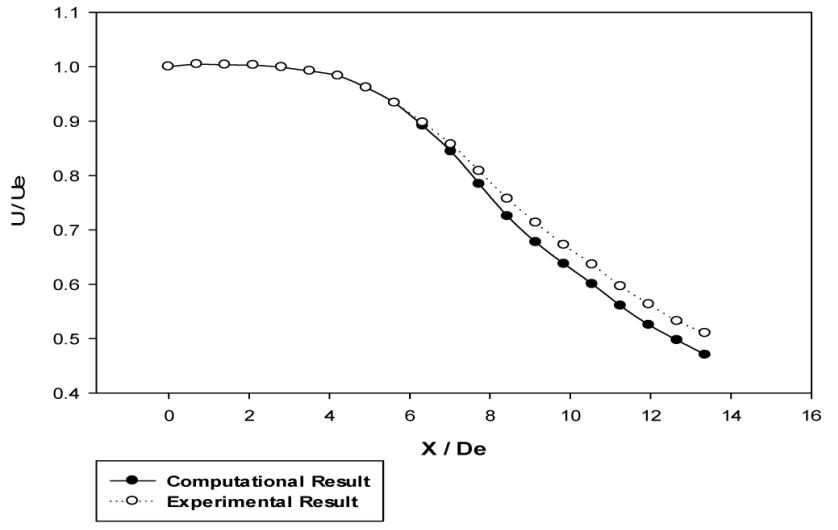

Figure 12. Centerline velocity decay of the jet with Groove in Minor Axis.

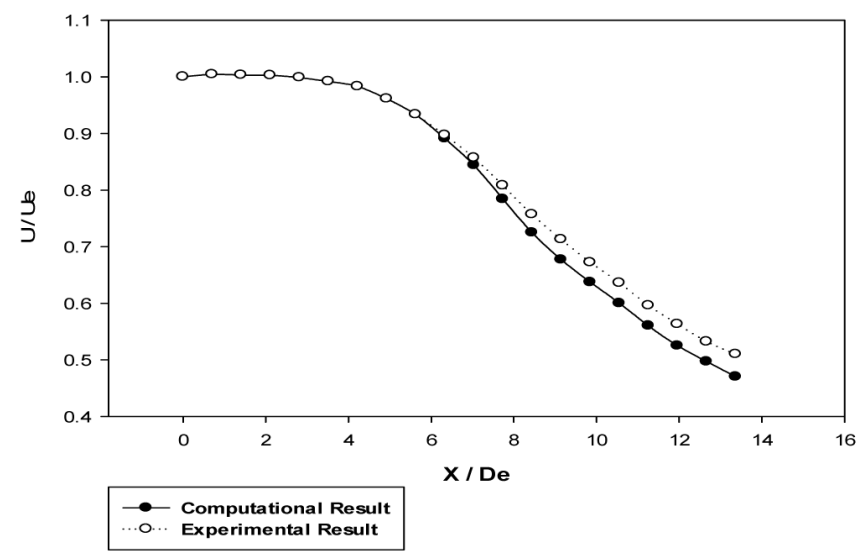

Figure 13. Centerline velocity decay of the jet with groove in both the axis.

core length only considerable difference in velocity distribution can be observed. It can be seen that computational results shows faster decay when compared with the experimental results. But the deviation is not significant; trend of both computational and experimental results are similar.

Figure 14 shows the centerline velocity decay for the jets from plain and grooved nozzles obtained from Computational results. It is observed that the potential core-length of plain jet and jet with grooves in major axis direction extends up to $3 \cdot 9 \mathrm{D}_{\mathrm{e}}$. On the other hand for jets with grooves in minor-axis direction and in both axes, the potential core-length extends up to $4 \mathrm{D}_{\mathrm{e}}$. The presence of the grooves in Minor axis direction and in both axes direction reduces mixing hence decrease in jet centerline velocity decay can be observed (increase in potential core-length). 


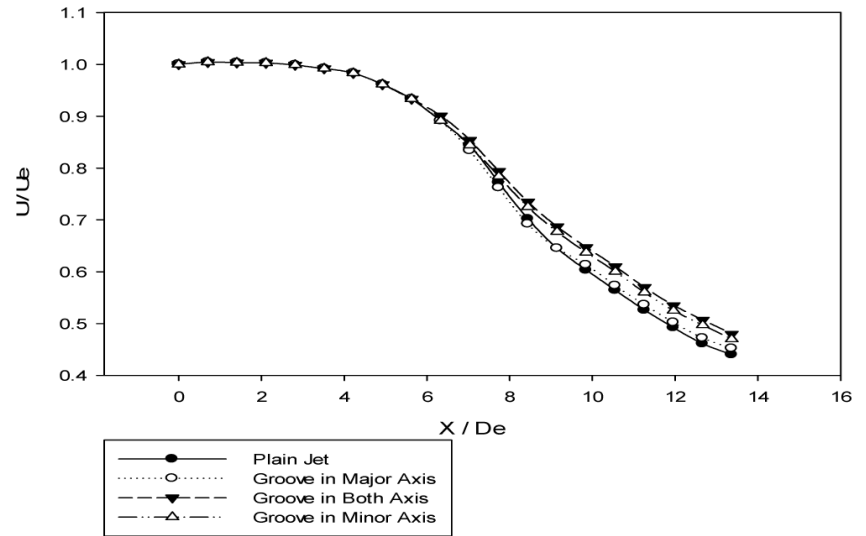

Figure 14. Centerline velocity decay of the jet for all the configurations (Computational).

\section{Conclusions}

A Computational Analysis has been carried out to study the flow development from a jet issuing from a 2:1 rectangular nozzle with and without grooves. Square grooves of side $4 \mathrm{~mm}$ and length $5 \mathrm{~mm}$ were separately introduced in the minor-axis direction, major-axis direction and on both the axes direction of rectangular nozzle.

It is observed that grooves significantly influence the jet growth beyond the potential core region. For jets with grooves in minor axis direction and in both axes, the potential core-length extends up to $4 \mathrm{D}_{\mathrm{e}}$. Presence of the grooves in minor axis direction and in both axes direction reduces mixing and hence decreases in jet centerline velocity decay also.
Computational Results also found to be good agreement with the experimental results.

\section{Acknowledgements}

The authors wish to acknowledge the assistance of Mr. Cholarajan T. J., Dilli Kannan G and John Jebaraj D., Graduate students of Rajalakshmi Engineering College, Chennai for the computation.

\section{References}

1. Ho C M, and Gutmark E J (1987). Vortex induction and mass entrainment in a small-aspect ratio elliptic jet, Journal of Fluid Mechanics, vol 179, 383-405.

2. Hussain H S, and Husain A K M F (1989). Elliptic jets part I: characteristics of unexcited and excited jets, Journal of Fluid Mechanics, vol 208, 257-319.

3. Verma S B, and Rathakrishnan E (1998). Mixing enhancement and noise attenuation in notched elliptic-slot jets, International Journal of Turbo and Jet Engines, vol 15, 7-25.

4. Srinivasan K, and Rathakrishnan E (1985). Studies on polygonal slot jets, AIAA Journal, vol 38, No. 10.

5. Elangovan S, and Rathakrishnan E (2004). Studies on high speed jets from nozzles with internal grooves, The Aeronautical Journal, vol 108, 43-50.

6. Arunkumar P, Verma S B et al. (2011). Study of jets from rectangular nozzles with square grooves, The Aeronautical Journal, vol 115, 187-196.

7. Pao S P, and Abdol K S (1996). Numerical simulation of jet aerodynamics using the three-dimensional Navier-stokes code PAB3D, NASA Technical Paper, NASA TP-3596, 1-43. 